\title{
SOME TAUBERIAN PROPERTIES OF HÖLDER TRANSFORMATIONS. ADDENDUM
}

\section{AMNON JAKIMOVSKI}

Theorem $\mathrm{C}$ of my paper mentioned above, in Proceedings of the American Mathematical Society vol. 7 (1956) pp. 354-363, was not quoted correctly and therefore the proof of Theorem 3.1 is not complete.

In the corrected statement of Theorem $\mathrm{C}$ it is necessary to add to the suppositions of Theorem $\mathrm{C}$ the supposition that the function $\alpha(t)$ is of regular bounded variation too.

A function of bounded variation is of regular bounded variation if it is the sum of its discontinuity function and an absolutely continuous function.

In order to complete the proof of Theorem 3.1 it is necessary to show that the function

$$
\begin{array}{r}
T(z)=(a \alpha+b \beta) \alpha^{-1}[1-(z+1)]^{-1}\left[1-a(z+1)^{-\alpha}-b(z+1)^{-\beta}\right], \\
\operatorname{Re} z \geqq 0,
\end{array}
$$

of Lemma 3.1 is a Mellin transform of a function of regular bounded variation in $0 \leqq t \leqq 1$. For this end it is sufficient to show that the function $\alpha(t)$ of Lemma $A$ is an absolutely continuous function in $0 \leqq t \leqq 1$ since for $n=0,1,2, \cdots, 1=\int_{0}^{1} t^{n} d \chi(t)$ where $\chi(1)=1, \chi(t)=0$ for $0 \leqq t<1$.

We have for $\alpha, \beta>0$ and $k$ an arbitrary positive integer

$$
\begin{aligned}
\frac{\beta}{\alpha} \frac{1-(z+1)^{-\alpha}}{1-(z+1)^{-\beta}} & =\frac{\beta}{\alpha}\left[1-(z+1)^{-\alpha}\right] \sum_{p=0}^{k}(z+1)^{-k \beta} \\
& +(\alpha z)^{+1}\left[1-(z+1)^{-\alpha}\right] \frac{\beta z}{(z+1)^{k \beta}\left[(z+1)^{\beta}-1\right]}
\end{aligned}
$$

$\left((z+1)^{-\alpha}=e^{-\alpha \cdot \log (z+1)}\right.$, where we choose for $\log (z+1)$ its principal branch). If $k$ is sufficiently large then by the proof of Lemma (5.4) of [1]

$$
\frac{\beta z}{(z+1)^{k \beta}\left[(z+1)^{\beta}-1\right]}=\int_{0}^{1} t^{z} \phi(t) d t \quad \text { for } \operatorname{Re} z \geqq 0
$$

where $\phi(t)$ is $L$-integrable in $0 \leqq t \leqq 1$. We also have (compare Theorem (8.1) of [1])

Received by the editors September 26, 1956. 
(3)

$$
(\alpha z)^{-1}\left[1-(z+1)^{-\alpha}\right]
$$

$$
=\int_{0}^{1} t^{z}[t \Gamma(\alpha+1)]^{-1}\left[\int_{0}^{t}\left(\log \frac{1}{u}\right)^{\alpha-1} d u\right] d t, \quad \operatorname{Re} z \geqq 0,
$$

(4) $(z+1)^{-\delta}=\int_{0}^{1} t^{2}[\Gamma(\delta)]^{-1}\left(\log \frac{1}{t}\right)^{\delta-1} d t, \quad \delta>0, \operatorname{Re} z \geqq 0$.

It is known (see $\left[3\right.$, p. $349, \S 15$, (1) with $s(u)=u^{z}$ and (2)]) that if

$$
\begin{array}{ll}
g(z)=\int_{0}^{1} t^{z} d \phi(t), & \operatorname{Re} z \geqq 0, \phi(t) \text { is of bounded variation, } \\
h(z)=\int_{0}^{1} t^{z} d \psi(t), & \operatorname{Re} z \geqq 0, \psi(t) \text { is of bounded variation, }
\end{array}
$$

then

$$
g(z) h(z)=\int_{0}^{1} t^{z} d \eta(t), \quad \operatorname{Re} z \geqq 0,
$$

where

$$
\eta(t)=\int_{t}^{1} \phi\left(\frac{t}{\tau}\right) d \psi(\tau)+\phi(1)[\psi(t)-\psi(0)], \quad 0 \leqq t \leqq 1,
$$

(the last integral is a Lebesgue-Stieltjes integral). If $\psi(t)$ is absolutely continuous then $\eta(t)$ defined by (5) is also absolutely continuous; hence (1), (2), (3), (4) and (5) show that the function which appears on the left side of (1) is a Mellin transform of an absolutely continuous function in $0 \leqq t \leqq 1$; the fact which completes the proof of Theorem 3.1 .

\section{University OF TeL-Aviv}

